\title{
RESEARCH HIGHLIGHT OPEN Structural basis of signaling of cannabinoids receptors: paving a way for rational drug design in controling mutiple neurological and immune diseases
}

\author{
Shenming Huang ${ }^{1,2}$, Peng Xiao ${ }^{1}$ and Jinpeng Sun ${ }^{1,2}$ \\ Signal Transduction and Targeted Therapy (2020)5:127
}

\author{
; https://doi.org/10.1038/s41392-020-00240-5
}

Cannabinoids (CBs), analgesic drugs used for thousands of years, were first found in Cannabis sativa, and the multiple CBs used medicinally, such as tetrahydrocannabinol (THC), cannabidiol (CBD) and dozens more, have complex structures. In addition to their production by plants, CBs are naturally present in the nerves and immune systems of humans and animals. Both exogenous and endogenous CBs carry out a variety of physiological functions by engaging with two $C B$ receptors, the $C B 1$ and $C B 2$ receptors, in the human endocannabinoid system (ECS). Both $C B 1$ and $C B 2$ are $G$ protein-coupled receptors that share a 7-transmembrane (7TM) topology. CB1, known as the central CB receptor, is mainly distributed in the brain, spinal cord, and peripheral nervous system. CB1 activation in the human body typically promotes the release of neurotransmitters, controls pain and memory learning, and regulates metabolism and the cardiovascular system. Clinically, CB1 is a direct drug target for drug addiction, neurodegenerative diseases, pain, epilepsy, and obesity. Unlike the exclusive expression of CB1 in the nervous system, CB2 is mainly distributed in peripheral immune cells. Selective CB2 agonists would have therapeutic potential in the treatment of inflammation and pain and avoid side effects caused by currently used clinical drugs. Although significant progress has been made in developing agonists toward $C B$ receptors, efficient clinical drugs targeting $C B$ receptors remain lacking due to their complex signaling mechanisms. The recent structural elucidation of CB receptors has greatly aided our understanding of the activation and signal transduction mechanisms of $\mathrm{CB}$ receptors.

Structural characterization of $C B$ receptors at the atomic level began in 2016, when Professor Zhi-jie Liu's laboratory and Dr. Zhenhua Shao in the Rosenbuam laboratory solved the crystal structure of CB1. ${ }^{1,2}$ This structural information greatly facilitated the understanding of CB1 ligand recognition and signal transduction mechanisms. Continuing this progress, Professor Zhi-jie Liu's laboratory determined two agonist-bound CB1 crystal structures, which not only uncover the agonist- $C B 1$ interactions within the orthosteric ligand-binding pocket but also disclose the different structural features of agonist-bound and antagonist-bound CB1. In 2019, Brian Kobilka's group and Skiniotis's group reported the cryo-electron microscopy structure of $\mathrm{CB} 1$ bound to an agonist, FUB, and downstream heterotrimeric Gi protein. The agonist, FUB, exhibited a high affinity for the orthostatic ligand-binding pocket of the $C B 1$ receptor, maintaining the $C B 1$ receptor in an active configuration to form a stable complex with nucleotide-free heterotrimeric $\mathrm{Gi}$ protein. The highly conserved orthosteric binding pocket of $\mathrm{CB} 1$ poses a great challenge for the rational drug design of potent CB1 agonists with high selectivity. Therefore, avoiding the orthosteric site and developing allosteric regulators of $C B 1$ have become $C B 1$ research hotspots. To address this issue, a collaborative effort by the teams of Dr. Shao Zhenhua and Dr. Rosenbuam resulted in solution of the crystal structure of CB1 in complex with an allosteric ligand. ${ }^{3}$ In this structure, the allosteric modulator ORG27569 was identified at the outside of the 7TM bundle of the receptor, buried in the cell membrane. This new discovery undoubtedly provides a new route for drug development toward the $\mathrm{CB} 1$ receptor.

Along with progress made in $C B 1$ research, the study of another $\mathrm{CB}$ receptor, $\mathrm{CB} 2$, has also achieved great breakthroughs. In 2019, Professor Zhi-jie Liu's laboratory solved the crystal structure of CB2 in complex with a rationally designed antagonist AM10257. This structure reveals the distinct antagonist-binding mode in CB2 and provides the molecular basis for the highdegree subtype selectivity of antagonists between CB1 and CB2. In January 2020, due to their combined efforts, the groups of Huaqiang $\mathrm{Xu}$, Xiangquan Xie, and Cheng Zhang published the three-dimensional structure of CB2 bound to the agonist WIN 55212-2 and heterotrimeric Gi protein, ${ }^{4}$ revealing the mechanisms by which the specific agonist WIN 55212-2 activates CB2 and CB2 interacts with the Gi protein. In the same issue of Cell, Zhi-jie Liu's group reported a systematic study on the structures of both $C B 1$ and $C B 2$ engaged with $G$ proteins, ${ }^{5}$ and the crystal structure of agonist-bound $C B 2$. By simultaneously solving the three-dimensional structures of the AM12033-CB2-Gi and AM841-CB1-Gi complexes, they revealed the structural basis for the activation of $\mathrm{CB} 1$ and $\mathrm{CB} 2$, as well as their coupling to downstream $G$ proteins.

CB1 and CB2 share 44\% sequence homology and are simultaneously activated by many natural $C B$ molecules. Consistently, recent structures of $C B$ receptors have provided the structural basis for this phenomenon and shown that these two receptors share very similar ligand-binding pockets at orthosteric sites, generating great challenges in the design of selective agonists. However, structural identification of the

\footnotetext{
'Department of Biochemistry and Molecular Biology, School of Basic Medical Sciences, Key Laboratory Experimental Teratology of the Ministry of Education, Cheeloo college of Medicine, Shandong University, 250012 Jinan, Shandong, China and 2Department of Physiology and Pathophysiology, School of Basic Medical Sciences, Key Laboratory of Molecular Cardiovascular Science of the Ministry of Education, Peking University, 100191 Beijing, China

Correspondence: Jinpeng Sun (sunjinpeng@sdu.edu.cn)

These authors contributed equally: Shenming Huang, Peng Xiao
}

Received: 18 May 2020 Accepted: 24 June 2020

Published online: 21 July 2020 

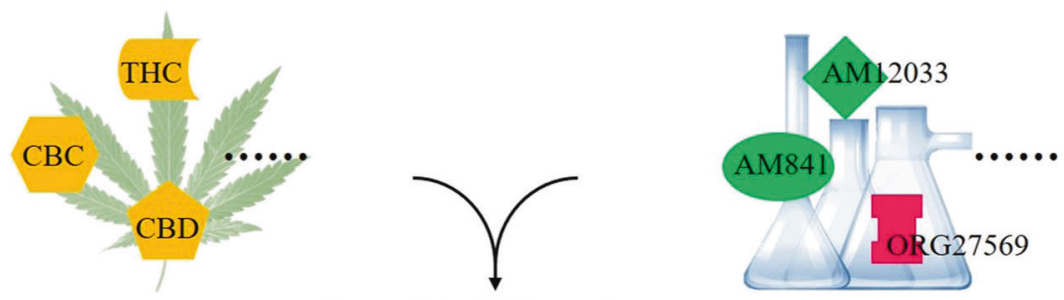

Cannabinoid Receptors

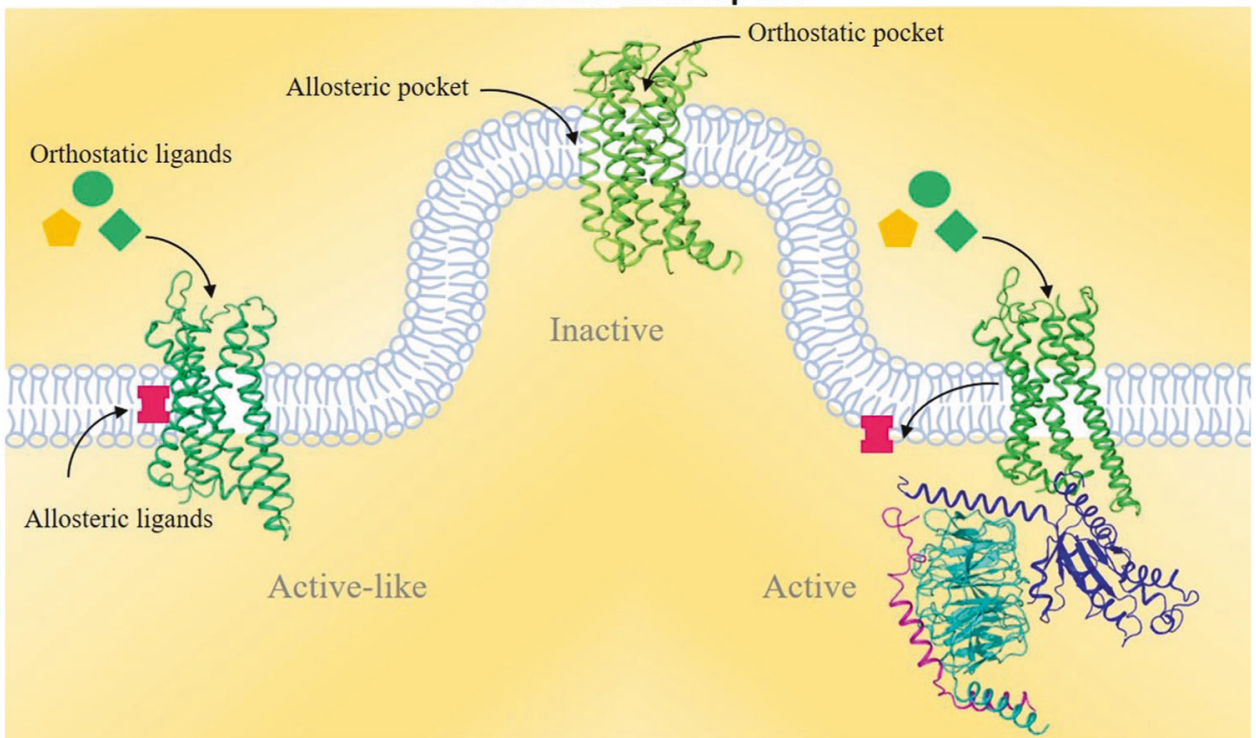

Fig. 1 Structural understanding of Cannabinoid receptors. Recent structural studies have revealed that two cannabinoid receptors (CB1 and CB2) shared a conserved orthostatic binding pocket for their agonists. Notably, an extra allosteric binding pocket was found for CB1 receptor. Both endogenous molecule cholesterol or synthetic ligand ORG27569 was able to bind to allosteric pockets, thus regulate activation state of CB1. Collectively, crystallographic and Cryo-EM studies have identified at least three structural states for CB receptors, which are inactive, active-like (intermediate) and active, indicating complex mechanisms underlying CB receptors' activation and signaling transduction.

allosteric ligand-binding sites in $\mathrm{CB}$ receptors provides new hope for the development of small compounds to selectively modulate $C B$ receptor functions. These recent structural studies suggest that $C B$ receptors adopt at least three states, an antagonistic state (inactive), intermediate state (active-like) and active state, which serves as the structural basis for complex signaling downstream of $C B$ receptors (Fig. 1). Recent structural characterizations of $C B$ receptors will greatly facilitate the design of new ligands to modulate the selective functions of $C B$ receptors. Notably, the CBD was approved by the Food and Drug Administration (FDA) in 2018 to treat epilepsy. We now look forward to more drugs targeting these two $C B$ receptors for clinical usage in the near future.

\section{ADDITIONAL INFORMATION}

Competing interests: The authors declare no competing interests.

\section{REFERENCES}

1. Shao, Z. et al. High-resolution crystal structure of the human CB1 cannabinoid receptor. Nature 540, 602-606 (2016).
2. Hua, T. et al. Crystal structure of the human cannabinoid receptor CB1. Cell 167, 750-762 e714 (2016).

3. Shao, Z. et al. Structure of an allosteric modulator bound to the CB1 cannabinoid receptor. Nat. Chem. Biol. 15, 1199-1205 (2019).

4. Xing, C. et al. Cryo-EM structure of the human cannabinoid receptor CB2-Gi signaling complex. Cell 180, 645-654 e613 (2020).

5. Hua, T. et al. Activation and signaling mechanism revealed by cannabinoid receptor-Gi complex structures. Cell 180, 655-665 e618 (2020).

(i) Open Access This article is licensed under a Creative Commons Attribution 4.0 International License, which permits use, sharing, adaptation, distribution and reproduction in any medium or format, as long as you give appropriate credit to the original author(s) and the source, provide a link to the Creative Commons license, and indicate if changes were made. The images or other third party material in this article are included in the article's Creative Commons license, unless indicated otherwise in a credit line to the material. If material is not included in the article's Creative Commons license and your intended use is not permitted by statutory regulation or exceeds the permitted use, you will need to obtain permission directly from the copyright holder. To view a copy of this license, visit http://creativecommons. org/licenses/by/4.0/.

(c) The Author(s) 2020 